\title{
Caracterização de um Sistema Convectivo de Mesoescala por meio de Sistema de Informações Geográficas
}

\author{
Characterization of a mesoscale convective system using a Geographic Information System \\ Robson Barreto dos Passos, Guilherme Batista Preisser, Michelle Simões Reboita, Enrique Vieira Mattos \\ Curso de Graduação em Ciências Atmosféricas no Instituto de Recursos Naturais da Universidade Federal de ltaubá.

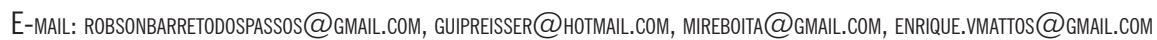

Abstract: Geographic Information System (GIS) is very popular for geography as well as environmental and hydric engineers, but less known in the atmospheric sciences. In this later, GIS can be an important tool for analyzes in climatology, synoptic meteorology, mesoscale meteorology, agrometeorology, atmosphere remote sensing and hydrometeorology. In this context, the present study has two purposes: (1) to classify the type of a mesoscale convective system that occurred in October 2017, among Paraguay, Brazil and Argentina, with the GIS Global Mapper15.0 and (2) to present a guide for the reader to reproduce the analyses presented here. The studied system caused great volumes of precipitation $(70 \mathrm{~mm} /$ day $)$ over Paraguay, Brazil and Argentina. Although visually the system was similar to the mesoscale convective complex, it was classified as a more elongated horizontal dimension system. During the maximum development of the convective system, the area and eccentricity were $719,044 \mathrm{~km}^{2}$ and 0.5 , respectively. The methodology presented in the study can be used to characterize intense systems in weather forecast model outputs, being useful in decision support by civil defense

\author{
Manuscrito: \\ Recebido: 21/08/2018
}

Aceito: $27 / 12 / 2018$

Citation: Passos, R. B. dos, Preisser, G. B., Reboita, M. S., Mattos E. V. 2019. Caracterização de um Sistema Convectivo de Mesoescala com uso de Sistema de Informação Geográfica. Terræ Didatica, 15, 1-10, e019010. doi: 10.20396/td.v15i0.8653308

Keywords: convective system, precipitation, Geographic Information System

\section{Introdução}

Os Sistemas Convectivos de Mesoescala (SCMs) são aglomerados de nuvens profundas que, em geral, possuem tempo de vida entre 6 e 12 horas (Houze, 1993). Os SCMs podem produzir precipitação contínua sobre regiões que excedem a $100 \mathrm{~km}$ na escala horizontal. Em contrapartida, as tempestades no interior dos $\mathrm{SCMs}$ podem produzir precipitação intensa, granizo, ventos fortes e relâmpagos (Houze, 1993; Cotton, Bryan \& Van Der Heever, 2010). O uso de Sistemas de Informação Geográfica como uma ferramenta para compreender as características físicas destes sistemas pode ser essencial no auxílio a tomadas de decisão pela defesa civil e governo local.

Os SCMs podem ser classificados conforme a sua forma geométrica em: Complexos Convectivos de Mesoescala (CCMs), Linhas de Instabilidade (LIs) e Sistemas Convectivos Alongados (SCAs). A origem dos SCMs pode ser tropical ou extratropical (Cotton \& Anthes, 1989). Este trabalho aborda um SCM extratropical.
A definição de CCM foi introduzida por Maddox (1980) ao analisar imagens de satélite no canal infravermelho em tempestades no centro dos Estados Unidos em 1978. Segundo o autor, os CCMs apresentam temperatura de brilho menor do que $-32^{\circ} \mathrm{C}$ numa área maior do que $100.000 \mathrm{~km}^{2}$ e, no interior dessa, possuem temperatura menor do que $-52^{\circ} \mathrm{C}$ numa área maior do que $50.000 \mathrm{~km}^{2}$. Além disso, devem possuir excentricidade maior que 0,7 no estágio de máximo desenvolvimento. O início do sistema ocorre quando os critérios de temperatura e área forem satisfeitos e sua duração é estabelecida quando esses mesmos critérios forem mantidos por mais de seis horas. Esses critérios garantem que o sistema seja ativo e que a precipitação ocorra sobre uma área significativa. Em suma, os CCMs são aglomerados de nuvens cumulonimbus que, em imagens de satélite, apresentam formato circular (Fig. 1a). A ocorrência de CCMs é comum na área entre Paraguai, norte da Argentina e Brasil (que constitui a Bacia do Prata). Nessa região, a combinação de alguns elementos meteo- 


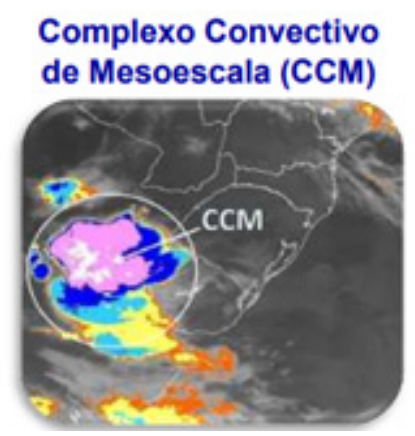

(a)

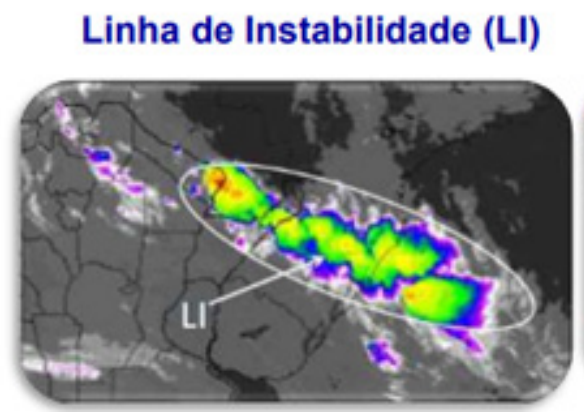

(b)

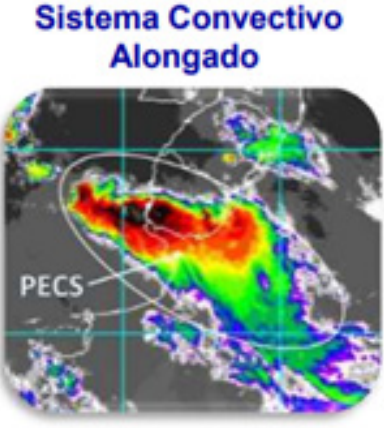

(c)

Figura 1. Sistemas Convectivos de Mesoescala em imagens realçadas de satélite do canal infravermelho: (a) Complexo Convectivo de Mesoescala, (b) Linha de Instabilidade e (c) Sistemas Convectivos Alongados. Adaptado de Fernandes (2010)

rológicos fornece o ambiente propício à formação dos CCMs, entre eles: o calor e umidade que são transportados da Amazônia para as latitudes subtropicais pelo jato de baixos níveis a leste dos Andes e a divergência de massa associada ao jato subtropical em níveis elevados da atmosfera (Silva Dias, 1987; Reboita, Gan, Rocha \& Ambrizzi, 2010; Cardoso Neta \& Silva, 2016). Entretanto, esses elementos também podem ser responsáveis pela gênese de outros SCMs sem forma definida.

As linhas de instabilidade (LIs) são bandas de nuvens cumulonimbus que se organizam em curva ou em linha (Fig. 1b), possuem um deslocamento uniforme no decorrer do seu ciclo de vida e teve duração de poucas horas até um dia (Houze, 1977; Cotton \& Anthes, 1989; Silva Dias, 1987). As LIs, em geral, se formam na interface entre uma massa de ar quente e úmida e uma massa de ar frio e seca e produzem ventos em superfície muito fortes (12$25 \mathrm{~m} / \mathrm{s}$ ) e, em alguns casos, até tornados. As LIs mais intensas estão relacionadas a linhas de células convectivas profundas, as quais surgem no setor quente, a cerca de 200-300 km adiante das frentes frias (Browning, 1986).

Sistemas Convectivos Alongados, em inglês conhecidos como Persistent Elongated Convective System (PECS), são sistemas convectivos formados por um aglomerado de nuvens cumulonimbus com formato alongado (Fig. 1c) e que também são responsáveis por precipitação intensa. Esses sistemas se enquadram nos critérios estabelecidos por Maddox (1980) quanto à duração e tamanho, mas se diferenciam dos CCMs pela excentricidade, por apresentarem valores menores do que 0,7 (Anderson \& Arritt, 1998).

Para classificar o tipo de um SCM é necessária a análise de uma sequência de imagens de satélite. Essa análise tem como objetivo estimar a área de atuação e excentricidade dos sistemas e pode ser realizada com a utilização de um Sistema de Informação Geográfica (SIG). O SIG pode ser definido como um sistema que integra tecnologia da informação, pessoas e informação geográfica, e cuja principal função é capturar, analisar, armazenar, editar e representar dados georreferenciados (Olaya, 2014). O uso do SIG tem aumentado nas últimas décadas em função do avanço tecnológico e por ter se tornado uma ferramenta acessível (Crampton, 2011).

Diversos estudos utilizam o SIG para espacializar informações (Wilhelmi \& Brunskill, 2003), como os de topografia, uso e ocupação do solo, precipitação entre outros. Por exemplo, Macedo et al. (2013) utilizaram o software ArcGis 9.2 para delimitação e cálculo das áreas de polígonos de Thiessen, a fim de caracterizar o regime de precipitação de uma dada região. Barbosa (2006) utilizou o SIG como ferramenta para análise e espacialização de dados climáticos, enquanto Ramos (1999), para a integração, distribuição espacial e visualização de parâmetros meteorológicos e oceanográficos. O uso do SIG na área de agrometeorologia é descrito por Sivakumar e Hinsman (2004), onde é mostrado como a combinação de dados de radar e satélite, modelos meteorológicos e cartografia digital podem facilitar o planejamento agrícola e a tomada de decisões. A aplicação do SIG em recursos hídricos e meio ambiente é bastante difundida, uma revisão acerca do uso dessas ferramentas na previsão de inundações, simulação de escoamento, mudanças hidrológicas e gerenciamento de recursos hídricos foi feita por Chen (2004). 
Embora o SIG seja muito difundido na geografia e engenharias ambiental e hídrica, em ciências atmosféricas é pouco conhecido e, sendo seu uso, muitas vezes, limitado à interpolação espacial de dados de precipitação. Portanto, o presente estudo é de grande importância para introduzir o SIG a novos usuários. Nesse contexto, o presente estudo tem dois objetivos: i) classificar o tipo de um sistema convectivo de mesoescala ocorrido, no período de 08 a 09 de outubro de 2017, entre o Paraguai, Brasil e Argentina, e que causou elevados totais de precipitação e ii) apresentar um roteiro para a classificação do sistema com o uso de um SIG, no caso, o Global Mapper 15.0.

\section{Metodologia}

O sistema em estudo (Fig. 2) se formou sobre o sul do Paraguai no dia 08 de outubro de 2017 e perdurou até o dia 09 , quando também atuou sobre a Argentina e Brasil.

As tonalidades em rosa e azul escuro na figura 2 sugerem que o sistema possui um formato quase circular podendo ser um CCM. Pela análise visual da imagem já está descartada a possibilidade do sistema ser LI. Diante disso, torna-se importante a avaliação acerca se o SCM é um CCM ou apenas um sistema com formato alongado. Para tanto, a metodologia consiste em calcular as variáveis da classificação de CCM proposta por Maddox (1980). Os critérios estabelecidos por esse autor são baseados na variável temperatura de brilho registrada pelos satélites no canal infravermelho $(10,7 \mu \mathrm{m}) \mathrm{e}$ estão apresentados na tabela 1.

Os critérios de Maddox (1980) foram aplicados em imagens de satélite obtidas do Centro de Previsão de Tempo e Estudos Climáticos (CPTEC; http://satelite.cptec.inpe.br/home/index.jsp) entre os dias 8 e 9 de outubro de 2017. Diversos horários foram analisados a fim de determinar as características do sistema ao longo do seu ciclo de vida. Entretanto, por questão de brevidade, aqui só será mostrado um tempo específico para descrição da utilização do SIG.

No intuito de avaliar a precipitação associada com o SCM, utilizaram-se dados diários obtidos do Climate Prediction Center (Chen et al., 2008) para os dias 08 e 09 de outubro. Esses dados possuem de resolução espacial de $0,5^{\circ}$ e cobrem apenas o continente. Uma análise adicional foi realizada para os relâmpagos totais associados com o sistema. Para isso, foram utilizadas as ocorrências de relâmpagos

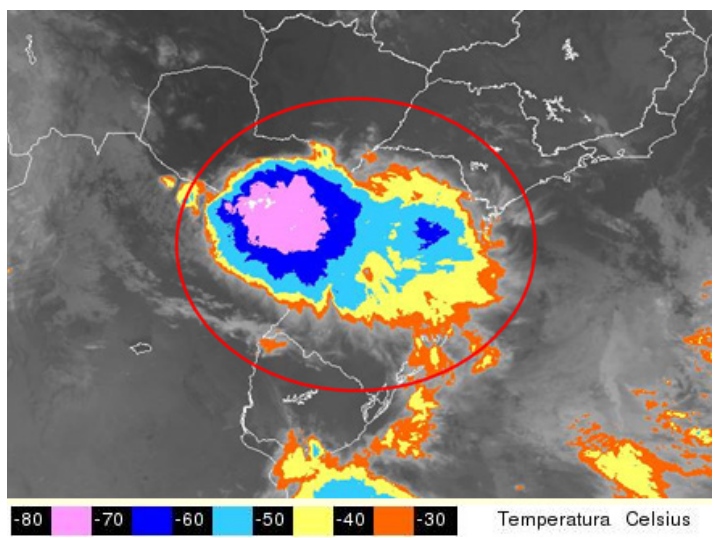

Figura 2. Imagem realçada do canal infravermelho (10,7 $\mu \mathrm{m})$ do satélite GOES-13 do dia 9 de outubro de 2017 às 06:30 UTC (03:30 horas local) quando o sistema atingiu a fase de máxima intensidade. Fonte: CPTEC/INPE

intra-nuvem e nuvem-solo da rede de relâmpagos da Earth Networks. Os sensores detectam as descargas de retorno por meio do método time-of-arrival (TOA) e são operados entre as frequências de 1 $\mathrm{Hz}$ e $12 \mathrm{MHz}$. (Naccarato \& Pinto, 2012). Para este estudo foram utilizados a data e a localização dos relâmpagos intra-nuvem e nuvem-solo. Estes dados foram fornecidos gentilmente pela empresa Climatempo.

Tabela 1. Critérios para a caracterização de um CCM baseado em Maddox (1980)

\section{Características físicas de um CCM}

(a) O sistema deve apresentar uma região de nebulosidade com temperatura no infravermelho $<-32^{\circ} \mathrm{C}$ abrangendo continuamente uma área $>$ $10^{5} \mathrm{~km}^{2}$

Tamanho: (b) Dentro da região delimitada em (a) deve haver uma região de nebulosidade com temperatura no infravermelho $<-52{ }^{\circ} \mathrm{C}$ abrangendo continuamente uma área $>5 \times 10^{4} \mathrm{~km}^{2}$.

\begin{tabular}{c|l}
\hline Inicio: & $\begin{array}{l}\text { Primeiro horário em que as condições } \\
\text { (a) e (b) são satisfeitas. }\end{array}$ \\
\hline Duração: & $\begin{array}{l}\text { As condições (a) e (b) devem ser satisfei- } \\
\text { tas por pelo menos } 6 \mathrm{~h} .\end{array}$ \\
\hline Formato: & $\begin{array}{l}\text { O sistema deve apresentar excentrici- } \\
\text { dade maior que } 0,7 \text { durante sua fase } \\
\text { madura. }\end{array}$ \\
\hline Término: & $\begin{array}{l}\text { Momento em que as condições (a) e (b) } \\
\text { não são mais satisfeitas. }\end{array}$
\end{tabular}




\section{Roteiro para determinar os critérios de Maddox (1980) utilizando o Global Mapper}

O Global Mapper é um SIG mantido pela empresa Blue Marble Geographics e com licença paga. No exemplo a seguir, é utilizada a versão 15.0 do software.

\section{Obtenção da imagem de satélite com o sistema atmosférico de interesse}

O formato da imagem de satélite pode ser JPEG ou GIF e pode ser obtida no website http://satelite. cptec.inpe.br/home/index.jsp. No exemplo, foi obtida a imagem do dia 09 de outubro de 2017 às 06:30 UTC do canal infravermelho $(10,7 \mu \mathrm{m})$ do satélite GOES-13.

\section{Carregando uma imagem de satélite no Global Mapper}

Quando se inicia o software visualiza-se a seguinte interface:

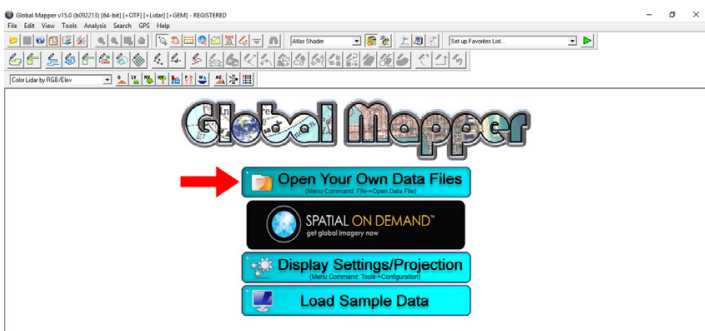

Figura 3. Interface inicial do software

Para abrir a imagem, é necessário clicar em "Open Your Own Data Files" (Fig. 3). Com isso uma janela se abrirá para selecionar a imagem no local em que ela está salva. Após este procedimento basta clicar em abrir e uma caixa de diálogo aparecerá (Fig. 4).

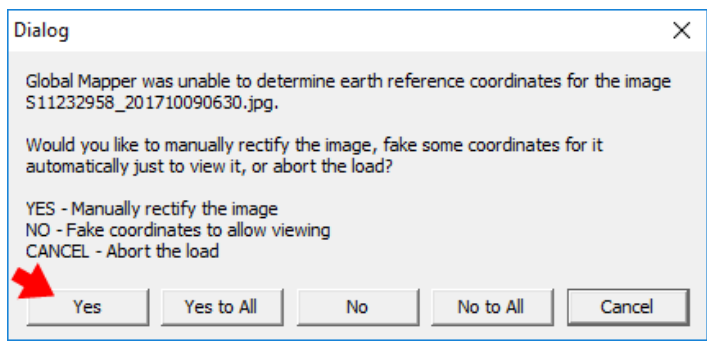

Figura 4. Caixa de diálogo sobre georreferenciamento da imagem
A caixa de diálogo está informa que a imagem não está georreferenciada, entretanto, para realizar as análises pretendidas é necessário que cada ponto da imagem possua as coordenadas geográficas (latitude e longitude) correspondentes.

\section{Georreferenciamento de uma imagem de satélite}

Georreferenciamento significa associar um sistema de coordenadas a uma imagem ou mapa. Para georreferenciar a imagem, inicie clicando em "Yes", como indicado na figura 4. A janela "Image Rectifier” se abrirá (Fig. 5). Nesta janela que se realiza o georreferenciamento da imagem.

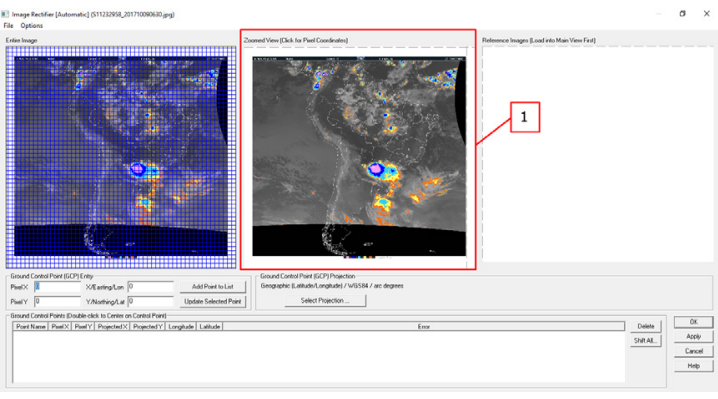

Figura 5. Janela "Image Rectifier" na qual se realiza o georreferenciamento da imagem

Para georreferenciar uma imagem, deve-se conhecer as coordenadas reais dos pontos das quatro extremidades da imagem. No caso desta imagem (GOES-13 obtida do site do CPTEC), as latitudes que a limitam são $12,52^{\circ} \mathrm{N}$ e $56^{\circ} \mathrm{S}$, enquanto que as longitudes são $100^{\circ} \mathrm{W}$ e $25,24^{\circ} \mathrm{W}$. Atenção: as imagens do GOES-16 fornecidas pelo mesmo site possuem limites diferentes.

Como exemplo, será feito a inserção das coordenadas do ponto superior esquerdo (ignorando o cabeçalho da imagem e a legenda no inferior dessa), que possui coordenada: latitude $12.52^{\circ} \mathrm{N}$, longitude $100^{\circ} \mathrm{W}$, para os outros pontos o procedimento é o mesmo.

Observe na marcação 1 da figura 5 que aparece o nome "Zoomed View". Essa janela permite ao usuário ampliar (exibir um zoom) um ponto desenhando retângulos sobre ele até ser possível visualizar seu pixel por inteiro (quanto mais próximo maior a precisão). Quando obtido o pixel, clique no seu centro que aparecerá um ponto vermelho (Marcação 2 na Fig. 6). 


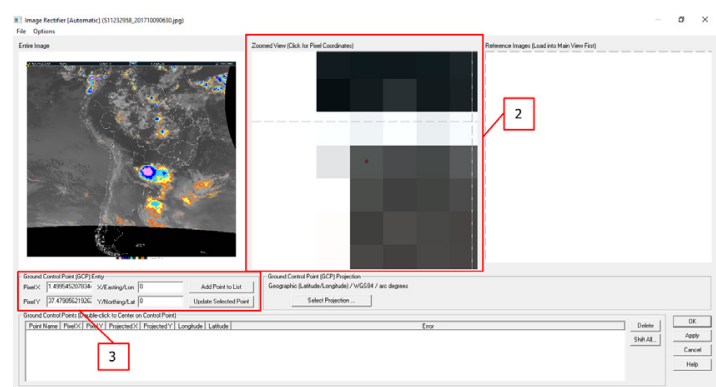

Figura 6. Detalhamento da janela "Image Rectifier" para o georreferenciamento da imagem

No ponto obtido é preciso informar suas coordenadas. Isso é feito na caixa de entrada "Ground Control Point Entry", destacada com a marcação 3 na figura 6 e ampliada na figura 7.

\begin{tabular}{|c|c|c|c|}
\hline \multicolumn{4}{|c|}{ Ground Control Point (GCP) Entry } \\
\hline PixelX & 1.499545207834 & $X /$ Easting/Lon $100^{\circ} \mathrm{W}$ & Add Point to List \\
\hline PixelY & $37.47905621926^{\prime}$ & Y/Northing/Lat $12.52^{\circ} \mathrm{N}$ & Update Selected Point \\
\hline
\end{tabular}

Figura 7. Caixa de entrada para inserção das coordenadas do ponto

Para inserir as coordenadas é importante saber as informações: Grau $=\left[^{\circ}\right]$, Minuto $=\left[^{\circ}\right]$, Segundo $=$ $["]$, Norte $=[\mathrm{N}]$, Leste $=[\mathrm{E}]$, Oeste $=[\mathrm{W}]$. Após digitar a coordenada, clica-se em "Add Point to list" (Fig. 7). O mesmo procedimento deve ser realizado para os outros três pontos. Com os quatro pontos georreferencia dos a tela aparece como na figura 8 :

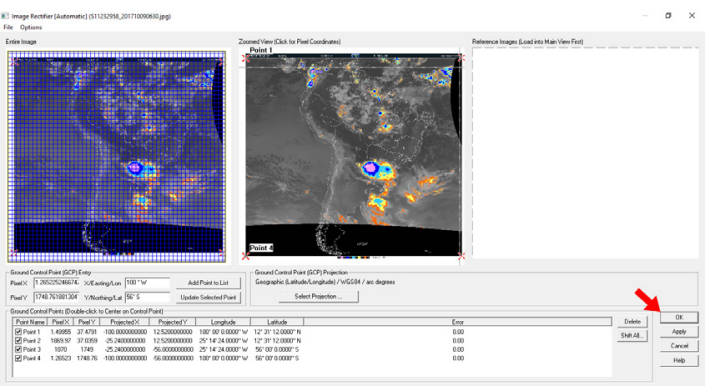

Figura 8. Janela "Image Rectifier" após a inserção das quatro coordenadas

Para terminar o georreferenciamento basta clicar em "OK” (Fig. 8). A imagem abrirá na tela principal do software onde serão realizadas as análises.

\section{Cálculo de Áreas}

Para computar a área de um objeto na imagem, inicialmente é necessário delimitá-la. Para isso, utiliza-se a ferramenta "Create new area feature".

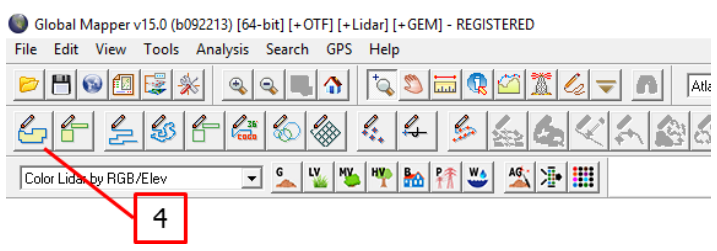

Figura 9. Ferramentas de análise do Global Mapper, com destaque para o ícone "Create new area feature"

Clicando no ícone "Create new area feature" (marcação 4 na Fig. 9), aparecerá um cursor que permite ao usuário desenhar (delimitar) um contorno na imagem (Marcação 5 na Fig. 10), a ferramenta de zoom é útil para uma melhor delimitação da região de interesse. Nesse exemplo, tem-se o objetivo de avaliar a classificação do SCM, seguindo os critérios de Maddox (1980), onde as temperaturas menores do que $-32{ }^{\circ} \mathrm{C}$ são delimitadas.

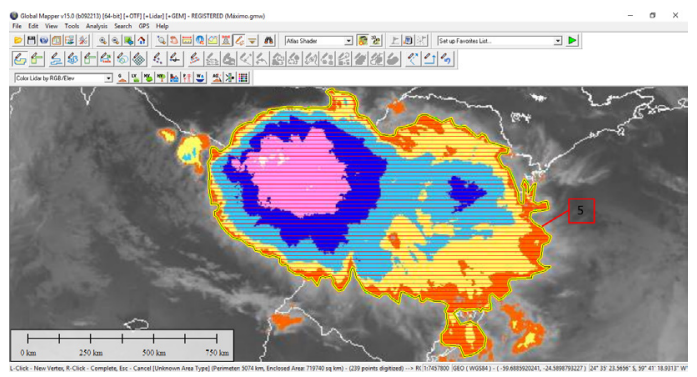

Figura 10. Delimitação da área de interesse

Após o término da delimitação, para fechar o contorno, basta um clique no botão direito do mouse. Nessa etapa, aparecerá uma nova janela que indica ao usuário a área e o perímetro da região delimitada (Fig. 11).

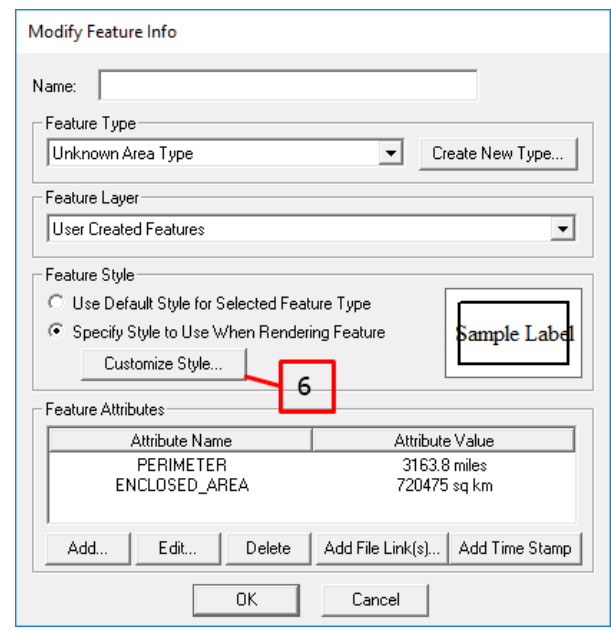

Figura 11. Janela com informações da área criada

\begin{tabular}{c|c|c|c|c|c}
\hline C Terrae Didat. & Campinas, SP & v.15 & $1-10$ & e019010 & 2019 \\
\hline \multicolumn{2}{|c}{5}
\end{tabular}


As características da área como cor e espessura do contorno podem ser modificadas habilitando-se a opção "Specify Style to Use When Rendering Feature" e depois clicando em "Customize Style" (Marcação 6 na Fig. 11), a janela mostrada na Figura 12 se abrirá para edição das características do contorno.

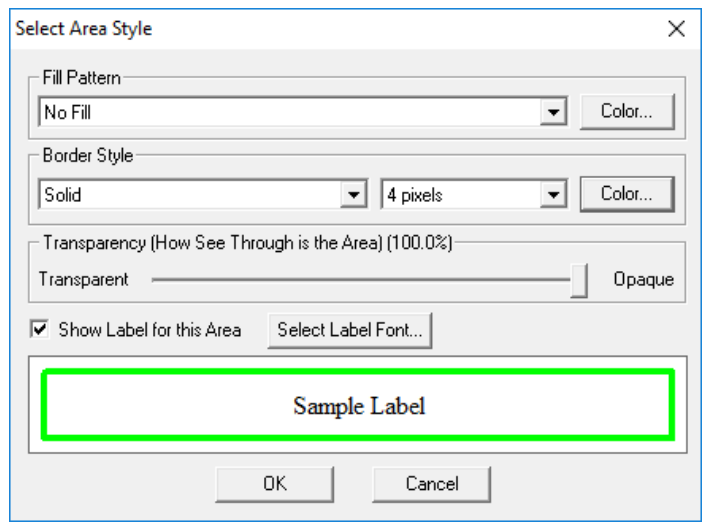

Figura 12. Janela para estilização do contorno criado

O Global Mapper trabalha com camadas (layers) de informação, então o usuário tem a opção de salvar as delimitações em um mesmo layer ou criar um layer para cada delimitação. Neste caso, será criado um layer específico para o contorno, como segue: seleciona-se a opção "Create New Layer for Feature" (Marcação 7 na Fig. 13).

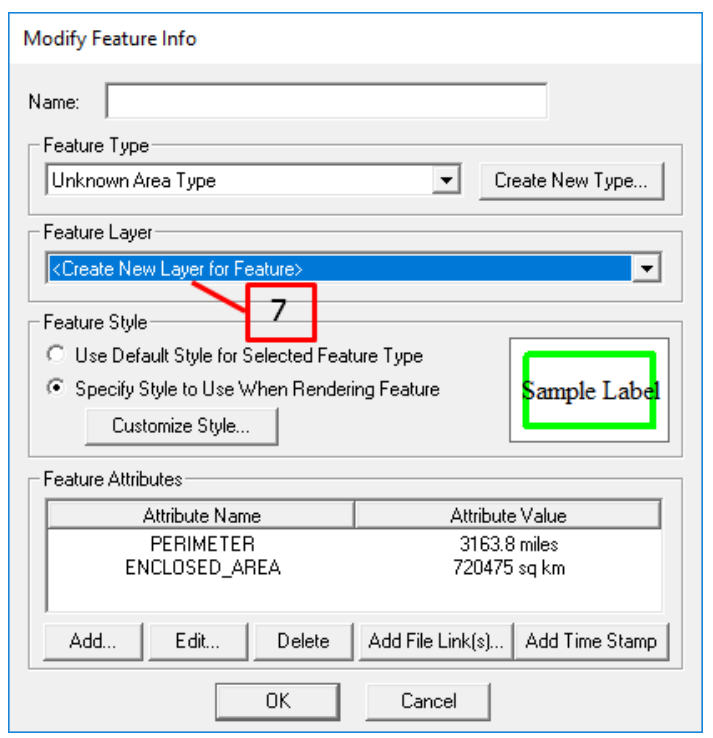

Figura 13. Criação de um novo layer para a área

Após clica-se em "OK". Por meio deste procedimento será aberta uma janela na qual será solicitado nomear o novo layer (Fig. 14).

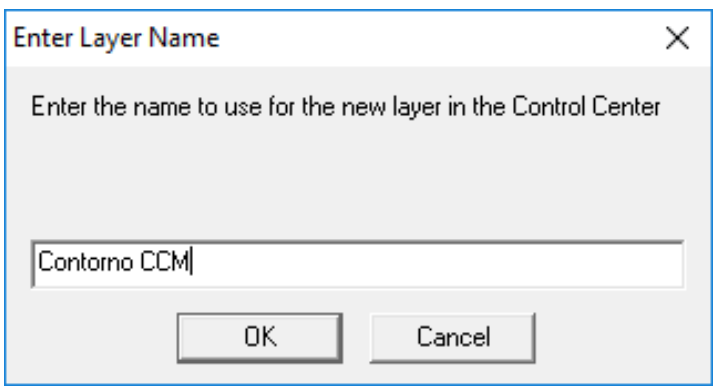

Figura 14: Janela para inserção do nome do novo layer

Após clicar em "Ok" (Fig. 14), o novo layer com a delimitação já estará definido. O controle da visualização pode ser realizado utilizando-se os layers, pela seleção do ícone "Open Control Center", como mostrado na figura 15.

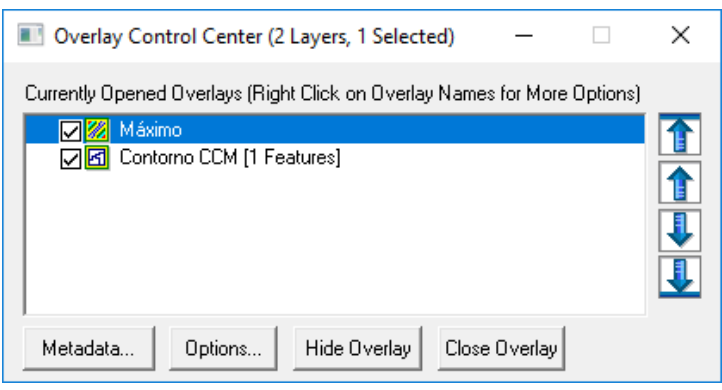

Figura 15. Painel de controle dos layers disponíveis

Realizando o mesmo procedimento, delimita-se, segundo os critérios de Maddox (1980), a área que apresenta temperatura menor que $-52^{\circ} \mathrm{C}$. A Figura 16 apresenta a imagem com as duas delimitações.

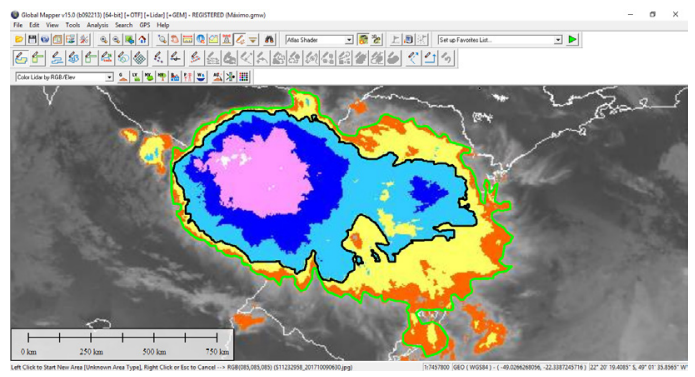

Figura 16. Imagem de satélite com as duas delimitações

A região com temperatura de brilho menor que $-32^{\circ} \mathrm{C}$ possui área de $719.044 \mathrm{~km}^{2}$, enquanto que a região com temperatura menor que $-52^{\circ} \mathrm{C}$ possui área de $436.586 \mathrm{~km}^{2}$. Comparando esses valores com os definidos por Maddox (1980), mostrados na Tabela 1, para caracterização de um CCM, verifica- 
-se que os critérios referentes às áreas e temperatura de brilho foram satisfeitos.

Um critério adicional definido por Maddox (1980) se refere à excentricidade. Para verificar essa característica do sistema será necessário traçar duas retas na área delimitada com temperatura menor do que $-32^{\circ} \mathrm{C}$ : uma reta para o eixo maior e outra para o eixo menor. O valor da excentricidade é obtido pela fórmula:

(Eq. 1):

Excentricidade $=\frac{(\text { comprimento do eixo menor })}{(\text { comprimento do eixo maior })}$

O valor obtido na equação 1 varia de 0 a 1 . Quanto mais próximo de 1, mais circular é o sistema e quanto mais próximo de 0 , o formato tende a ser elíptico.

Para facilitar a criação da reta é recomendável que se oculte a exibição do layer do contorno da região de $-52{ }^{\circ} \mathrm{C}$ utilizando o painel de controle mostrado na Figura 15. Para desenhar as duas retas utiliza-se a ferramenta "Create New Line Feature". Neste caso, o valor de excentricidade obtido foi de 0,5. A Figura 17 apresenta os dois eixos traçados:

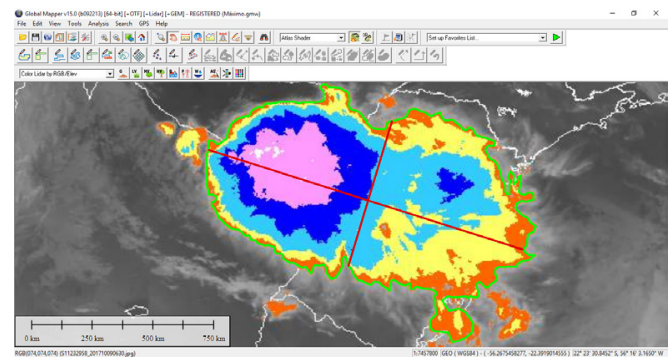

Figura 17. Imagem de satélite canal infravermelho (10,7 $\mu \mathrm{m}$ ) realçada indicando o eixo maior e menor (retas na cor vermelha) do sistema

\section{Resultados}

Após a aplicação da metodologia em diversas imagens de satélite, entre os dias 08 e 09 de outubro de 2017, foram selecionadas quatro imagens, que são as mais representativas do ciclo de vida do SCM (Fig. 18): i) o estágio anterior ao início do sistema, no qual os critérios do Maddox (1980) não foram satisfeitos (Fig. 18a), ii) o estágio de início do sistema no qual os critérios do Maddox foram observados pela primeira vez (Fig. 18b), iii) o estágio do máximo desenvolvimento do sistema, quando o sistema mostrou a maior área (Fig. 18c) e iv) o estágio de dissipação (Fig. 18d), quando nenhuma das duas áreas com maior dimensão presentes na Figura 18d satisfazem os critérios de Maddox (1980). As datas e os valores das áreas delimitadas na Figura 18 são apresentados na Tabela 2 .

O sistema teve tempo de vida de 17 horas, satisfazendo o critério da duração (Tab. 1), no qual as áreas devem permanecer maiores do que o limiar estabelecido por um período de pelo menos 6 horas (Maddox, 1980).
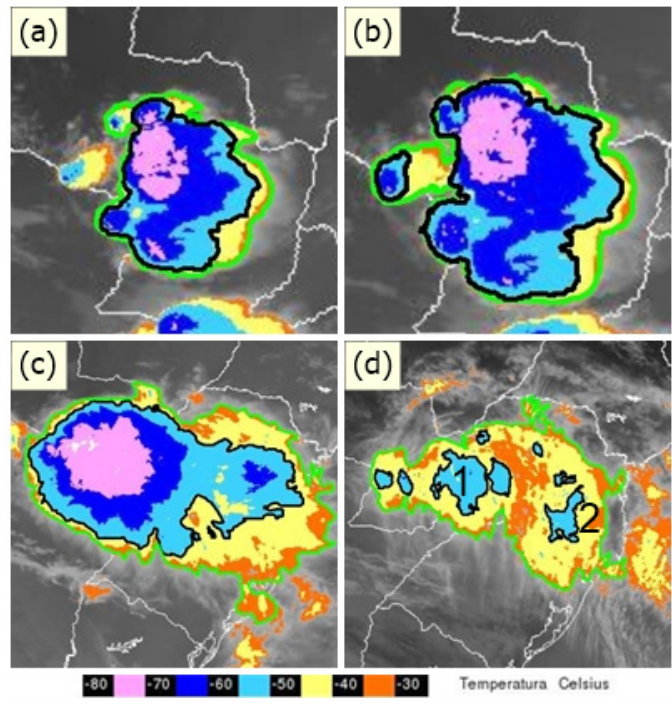

Figura 18. Imagens de satélite realçada do canal infravermelho para diferentes momentos do ciclo de vida do sistema avaliado: (a) Estágio antes da formação do sistema - 08/10/2017 21:30; (b) Estágio inicial do sistema - 08/10/2017 22:00; (c) Estágio do máximo desenvolvimento do sistema - 09/10/2017 06:30; (d) Estágio de dissipação do sistema - 09/10/2017 14:30. Contorno verde delimita a região que abrange a temperatura de $-32^{\circ}$ $\mathrm{C}$ e contorno preto delimita a região que abrange a temperatura de $-52{ }^{\circ} \mathrm{C}$

Maddox (1980) menciona que, para o cálculo das áreas, os núcleos de temperatura do sistema devem ser contínuos, ou seja, não podem ser fragmentados. Verifica-se que tanto a região com temperatura menor que $-32{ }^{\circ} \mathrm{C} \mathrm{e}-52{ }^{\circ} \mathrm{C}$ são contínuas nos estágios inicial e de máxima intensidade (Fig. 18b-c); entretanto, no período de dissipação (Fig. 18d), há presença de vários núcleos distintos de temperatura menor do que $-52^{\circ} \mathrm{C}$. Nesse último estágio, os critérios de Maddox (1980) foram avaliados separadamente para as duas maiores áreas e essas estão classificadas como área 1 e área 2 na figura $18 d$. 
Tabela 2. Valores das áreas calculadas utilizando o Global Mapper

\begin{tabular}{|c|c|c|c|}
\hline Estágios do sistema & Horário (UTC) & $\begin{array}{l}\text { Área com temperatura }< \\
\qquad-32^{\circ} \mathrm{C}\left(\mathrm{km}^{2}\right)\end{array}$ & $\begin{array}{l}\text { Área com temperatura }< \\
\qquad-52{ }^{\circ} \mathrm{C}\left(\mathrm{km}^{2}\right)\end{array}$ \\
\hline Antes & 08/10/2017 21:30 & $97.534 \mathrm{~km}^{2}$ & $74.400 \mathrm{~km}^{2}$ \\
\hline Inicio & 08/10/2017 22:00 & $125.544 \mathrm{~km}^{2}$ & $95.265 \mathrm{~km}^{2}$ \\
\hline Máximo & 09/10/2017 06:30 & $719.044 \mathrm{~km}^{2}$ & $436.586 \mathrm{~km}^{2}$ \\
\hline Dissipação & 09/10/2017 14:30 & $355.194 \mathrm{~km}^{2}$ & $\begin{array}{l}\text { Área 1: } 38.213 \mathrm{~km}^{2} \\
\text { Área 2: } 13.951 \mathrm{~km}^{2}\end{array}$ \\
\hline
\end{tabular}

A excentricidade foi calculada para a área do sistema com temperatura menor do que $-32{ }^{\circ} \mathrm{C} \mathrm{e}$ o valor obtido foi de 0,5 . Segundo a classificação de Maddox (1980), a excentricidade deve ser igual ou maior que 0,7 para classificar um SCM como CCM. Portanto, por esse critério o sistema em estudo não se classifica na categoria de CCM e, sim, na de sistema mais alongado horizontalmente.

O SCM produziu grande quantidade de precipitação na fronteira entre Brasil, Argentina e Paraguai. A Figura 19 apresenta o acumulado de precipitação proveniente do Climate Prediction Center nos dias 08 e 09 de outubro de 2017. No dia 08/10/2017 (Fig. 19a), o acumulado de precipitação diário chegou a atingir $70 \mathrm{~mm}$ no dia 08/10/2017 na região oeste do Paraná e também chegou a esses valores elevados no dia 09/10/2017 nas regiões central e sul do Paraguai. A área com totais elevados de precipitação no dia 09 é maior do que a do dia 08 ; no dia 08 o SCM estava no seu estágio inicial, enquanto que no dia 9 no de máximo desenvolvimento, portanto, ocupando uma área maior. Durkee e Mote (2009) documentaram uma contribuição dos SCM em 15-21\% no total anual de precipitação nas regiões norte da Argentina e Paraguai. Estes resultados mostram a importância de se identificar e rastrear os SCMs visando determinar as suas características físicas por meio de ferramentas como o SIG.
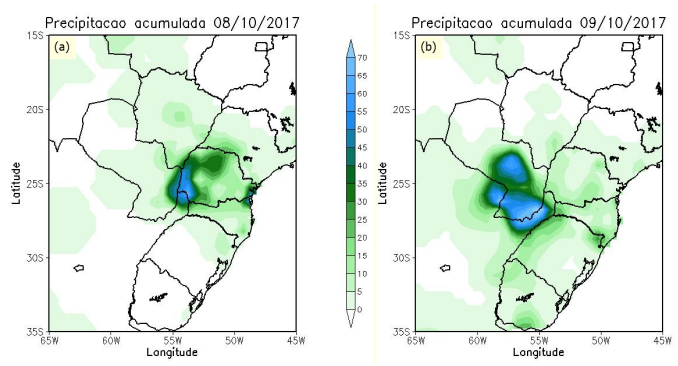

Figura 19. Precipitação acumulada $(\mathrm{mm})$ proveniente do CPC para os dias (a) 08/10/2017 e (b) 09/10/2017
Os SCMs são sistemas com grande desenvolvimento vertical e possuem fortes correntes ascendentes e produção de água líquida super-resfriada e partículas de gelo que, por sua vez, são características fundamentais para a formação de relâmpagos (Reynolds, Brook \& Gourley, 1957). As nuvens com grande desenvolvimento vertical, que constituem os SCMs, podem penetrar a tropopausa e injetar umidade na baixa estratosfera (Schmetz, Tjemkes, Gube \& Van de Berg, 1997; Machado, Lima, Pinto \& Morales, 2009). As nuvens podem ser identificadas por meio da técnica de multicanais, expressa pela diferença entre a temperatura de brilho do canal vapor d'água (TW, 6,5 $\mu \mathrm{m}$ ) e infravermelho (TIR, 10,7 $\mu \mathrm{m}$ ) (TWV-TIR) e as diferenças positivas estão associadas a topos de nuvens com overshooting.

A Figura 20 mostra o campo da diferença TWV-TIR (regiões coloridas) e ocorrência de relâmpagos totais (intra-nuvem + nuvem-solo) (cruz na cor vermelha). Alta incidência de relâmpagos totais é observada nas regiões com diferenças positivas de temperatura de brilho. Por exemplo, no estágio maduro, valores de até de $5 \mathrm{~K}$ são observados na porção oeste do sistema. Machado et al. (2009) utilizaram dados de satélite e relâmpagos da rede RINDAT e observaram um aumento nas ocorrências de relâmpagos, com o aumento das diferenças TWV-TIR (até $2 \mathrm{~K}$ ). Como discutido por Schmetz et al. (1997), as regiões com diferenças positivas são indicativos de fortes correntes ascendentes e que são capazes de umedecer a baixa estratosfera, condição que implica em maior temperatura no canal vapor d'água (localizado na estratosfera) do que no infravermelho (localizado no topo da troposfera). Estas regiões possuem alta quantidade de partículas de gelo, gotículas super-resfriadas e fortes correntes ascendentes, condições fundamentais para a eletrificação das nuvens (Reynolds et al., 1957). 

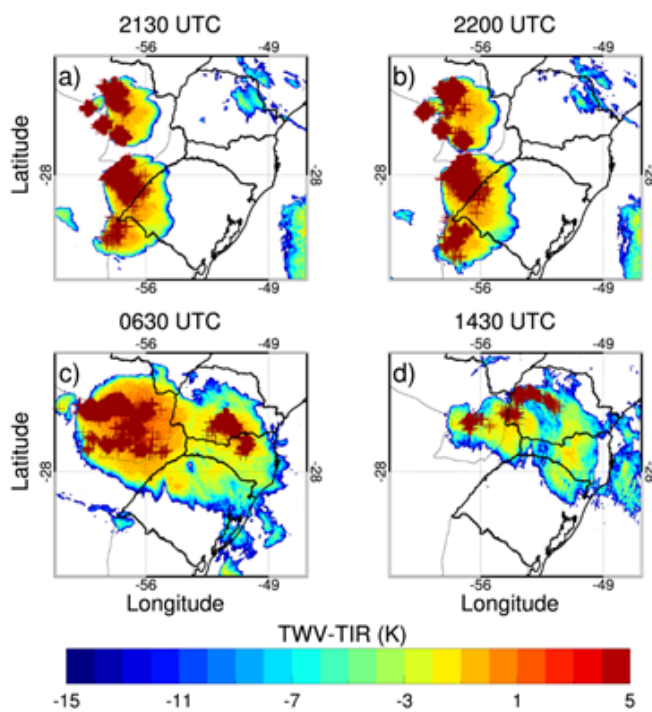

Figura 20. Diferenças de temperatura de brilho entre o canal do vapor d'água (TW, 6,5 $\mu \mathrm{m}$ ) e a temperatura de brilho no canal do infravermelho (TIR, 10,7 $\mu \mathrm{m}$ ) (TWV-TIR, região colorida) e ocorrências de relâmpagos totais (intra-nuvem e nuvem-solo) representados pelas cruzes na cor vermelha

\section{Conclusão}

O presente estudo teve como objetivo caracterizar por intermédio de Sistema de Informação Geográfica (SIG) um Sistema Convectivo de Mesoescala (SCM) que ocorreu entre os dias 08 e 09 de outubro de 2017 sobre o Paraguai, Argentina e Brasil. Para tanto, foram utilizadas de satélite do canal infravermelho do satélite GOES-13 e o software Global Mapper para avaliar o sistema, que produziu precipitação e relâmpagos. Este artigo apresenta a proposta de se introduzir o uso do SIG na área de ciências atmosféricas, área na qual tal ferramenta ainda é utilizada de maneira pontual.

O SCM produziu totais elevados de precipitação diária e uma alta frequência de relâmpagos totais, que foram associados a regiões de baixas temperaturas no interior do sistema. As áreas com temperaturas menores do que $-32^{\circ} \mathrm{Ce}-52^{\circ} \mathrm{C}$ satisfizeram os critérios propostos por Maddox (1980) para a definição de um clássico Complexo Convectivo de Mesoescala (CCM), o que permitiu definir os horários de início, máximo desenvolvimento e dissipação do sistema. Com isso, verificou-se que o sistema teve tempo de vida de 17 horas, satisfazendo o critério de duração (mínimo de 6 horas). No entanto, como o SCM apresentou excentricidade menor que o proposto para um CCM $(0,5$ versus 0,7), o mesmo foi classificado como SCM com dimensão horizontal mais alongada. A caracterização do SCM por meio de SIG mostrou-se satisfatória e de maior precisão nos detalhes da estrutura espacial do sistema em comparação com análises visuais de imagens de satélite ou por intermédio de classificação subjetiva.

O SIG mostrou-se importante no dimensionamento da área do sistema. A determinação do tamanho, excentricidade e inclinação de um sistema pode ser fundamental para se conhecer as características das nuvens que atuam sobre uma região. A utilização do SIG em conjunto com outras ferramentas de rastreamento e de estimativa de precipitação pode ser essencial para contribuir para a mitigação de desastres naturais associados à ocorrência destes sistemas. Tempestades de menor porte também podem ser importantes na produção de granizo, relâmpagos e ventos fortes. Portanto, o SIG é uma ferramenta que auxilia o estudo de sistemas atmosféricos com diferentes dimensões e sem restrição de localização espacial.

Por fim, destaca-se que a utilização do SIG em ciências atmosféricas também permitirá a integração com outras áreas do conhecimento, sendo, portanto, um elemento que motivará pesquisas conjuntas e poderá contribuir na melhoria da comunicação científica na área de ciências atmosféricas.

\section{Agradecimentos}

Os autores agradecem ao Centro de Previsão de Tempo e Estudos Climáticos (CPTEC) pelo fornecimento das imagens de satélite, ao Climate Prediction Center (CPC) pelos dados de precipitação, a Climatempo pelos dados de relâmpagos da Earth Network e ao Conselho Nacional de Desenvolvimento Científico e Tecnológico (CNPq) e à Fundação de Amparo à Pesquisa do Estado de Minas Gerais FAPEMIG pelo auxílio financeiro.

\section{Referências}

Anderson, C. J., \& Arritt, R. W. (1998). Mesoscale convective complexes and persistent elongated convective systems over the United States during 1992 and 1993. Monthly Weather Review, 126(3), 578-599. Disponível em: https://doi.org/10.1175/1 520-0493(1998)126<0578:MCCAPE > 2.0.CO;2

Barbosa, J. P. M. (2006). Utilização de método de interpolação para análise e espacialização de dados climáticos: o SIG como ferramenta. Caminhos de Geografia, 9(17), 85-96. 
Browning, K. A. (1986). Conceptual models for precipitation systems. Weather and Forecasting, 1, 23-41. Disponível em: https://doi. org/.0.1175/1520-0434(1986)001<0023:CMOPS $>2.0 . \mathrm{CO} ; 2$

Cardoso Neta, L., \& Silva, M. V. (2016). Análise de um Sistema Convectivo no Sul do Brasil Utilizando Índices de Instabilidade. Ciência e Natura, 38(1). Disponível em: https://doi.org/10.5902/2179460X14853

Chen, M., Shi, W., Xie, P., Silva, V. B. S., Kousky, V. E., Wayne, H. R., \& Janowiak, J. E. (2008). Assessing objective techniques for gauge-based analyses of global daily precipitation. Journal of Geophysical Research, 113, 1-13. Disponível em: https://doi. org/10.1029/2007JD009132

Chen, Y. (2004). GIS and remote sensing in hydrology, water resources and environment (No. 289). Wallingford: IAHS press.

Cotton, R. W., \& Anthes, B. R. (1989). Storm and cloud dynamics. Cambridge: Academic Press.

Cotton, R. W., Bryan, G., \& Van Den Heever, S. C. (2010). Storm and cloud dynamics. Cambridge: Academic Press.

Crampton, J. W. (2011). Mapping: A critical introduction to cartography and GIS. New Jersey: John Wiley \& Sons.

Durkee, J. D., \& Mote, T. L. (2009). A climatology of warm-season Mesoscale Convective complexes in subtropical South America. Int. J. Climatol. 30(3), 418-431. Falta o Disponível em: https://doi. org/10.1002/joc.1893

Fernandes, D. S. (2010). Caracterização das Tempestades a partir dos canais Infravermelho e Vapor d'água do Satélite GOES 10 e 12. (Dissertação de mestrado). São Paulo, SP, Universidade de São Paulo,

Houze, R. B. (1977). Structure and dynamics of a tropical squall-line system. Monthly Weather Review, 105(12), 1540-1567. Disponível em: https://doi. org/10.1175/1520-0493(1977)105<1540:SADO$\mathrm{AT}>2.0 . \mathrm{CO} ; 2$

Houze, R. B. (1993). Cloud dynamics. San Diego: Academic Press.

Olaya, V. 2014. Sistemas de Información Geográfica. Disponível em: http://volaya.github.io/libro-sig/

Macedo, M. N. C., Dias, H. C. T., Coelho, F. M. G., Araújo, E. A., Souza, M. L. H., \& Silva, E. (2013). Precipitação pluviométrica e vazão da bacia hidrográfica do Riozinho do Rola, Amazônia Ocidental. Revista Ambiente e Água, 8(1), 206-221. Dispo- nível em: https://doi.org/10.4136/ambi-agua.809

Machado, L. A. T., Lima, W. F. A, Pinto, O., \& Morales, C. A. (2009). Relationship between cloudto-ground discharge and penetrative clouds: A multi-channel satellite application. Atmospheric Research, 93(1-3), 304-309. Disponível em: https:// doi.org/10.1016/j.atmosres.2008.10.003

Maddox, R. A. (1980). Mesoscale convective complexes. Bulletin of the American Meteorological Society, 61(11), 1374-1387.

Naccarato, K. P, \& Pinto, O. (2012). Lightning detection in Southeastern Brazil from the new Brazilian Total lightning network (BrasilDAT), In: 2012 Internation Conference on Lightning Protection (ICLP). Vienna.

Ramos, J. A. P. (1999). Uso de sistemas de informações geográficas (SIG) e banco de dados para análise de parâmetros meteorológicos e oceanográficos. Dissertação de mestrado, Instituto Nacional de Pesquisas Espaciais, São José dos Campos, SP, Brasil.

Reboita, M. S., Gan, M. A., Rocha, R. P., \& Ambrizzi, T. (2010). Regimes de precipitação na América do Sul: uma revisão bibliográfica. Revista Brasileira de Meteorologia, 25(2). Disponível em: https://doi. org/10.1590/S0102-77862010000200004

Reynolds, S. E., Brook, M., \& Gourley, M. F. (1957). Thunderstorm charge separation. Journal of $\mathrm{Me}-$ teorology, 14(5), 426-436. Disponível em: https:// doi.org/10.1175/1520-0469(1957)014<0426: T $\mathrm{CS}>2.0 . \mathrm{CO} ; 2$

Schmetz, J., Tjemkes, S. A., Gube, M., \& Van de Berg, L. (1997). Monitoring deep convection and convective overshooting with METEOSAT. Advances in Space Research, 19(3), 433-441. Disponível em: https://doi.org/10.1016/S0273-1177(97)00051-3

Silva Dias, M. A. F. (1987). Sistemas de mesoescala e previsão de tempo a curto prazo. Revista Brasileira de Meteorologia, 2(1), 133-150.

Sivakumar, M. V. K., Roy, P. S., Harmsen, K., \& Saha, S. K. (2004). Satellite remote sensing and GIS applications in agricultural meteorology. In: Proceedings of the Training Workshop, Dehra Dun, Índia.

Wilhelmi, O. V., \& Brunskill, J. C. (2003). Geographic information systems in weather, climate, and impacts. Bulletin of the American Meteorological Society, 84(10), 1409-1414. Disponível em: https://doi. org/10.1175/BAMS-84-10-1409 